\title{
Review: use of hormone replacement therapy in the past 5 years is associated with an increased risk of breast cancer
}

\author{
Collaborative Group on Hormonal Factors in Breast Cancer. Breast cancer and hormone replacement therapy: collaborative \\ reanalysis of data from 51 epidemiological studies of 52705 women with breast cancer and 108411 women without breast \\ cancer. Lancet 1997 Oct 11;350:1047-59.
}

\section{Question}

Is hormone replacement therapy (HRT) associated with an increased risk of breast cancer?

\section{Data sources}

Studies were identified from review articles, literature searches, and colleagues.

\section{Study selection}

Studies were selected if they included $\geqslant 100$ women with breast cancer and if information was obtained on the use of HRT and reproductive and menopausal history.

\section{Data extraction}

Data were collected from the investigators for individual women on their use of HRT and hormonal contraceptives, sociodemographic factors, family history of breast cancer, height, weight, age at menarche, reproductive history, gynaecological surgery, menopausal status, age at menopause, and tumour spread for those who had breast cancer.

\section{Main results}

51 studies from 21 countries involving 52705 women with invasive breast cancer and 108411 women without breast cancer were included ( $81 \%$ of eligible studies). Meta-analyses were stratified by study, study centre, age at diagnosis, time since menopause, body mass index, parity, and, when appropriate, age of the woman at first birth. The main analyses were based on 53865 postmenopausal women (17 949 with breast cancer and 35916 without breast cancer) with known age at menopause. The overall median age at first use of HRT was 48 years. The relative risk (RR) of breast cancer among women who had used HRT at some time compared with those who had never used it was $1.14(\mathrm{p}<0.001)$. Compared with women who had never used HRT, those who had used it for $\geqslant 5$ years had an increased risk (RR 1.35, 95\% CI 1.21 to $1.49, \mathrm{p}<0.001$ ); current users (women who had used HRT at the time of or within 12 months of the diagnosis of breast cancer) had an increased risk (RR 1.21, $\mathrm{p}<0.001$ ); and those who had stopped use $\geqslant 5$ years before diagnosis had no increase in risk. Among current users or those who had stopped use 1-4 years previously, the RR increased by a factor of 1.02 (CI 1.01 to $1.04, \mathrm{p}<0.001$ ) for each year of use. For women who used HRT $<5$ years before diagnosis and the duration of use was $\geqslant 5$ years, an increase in the risk of breast cancer was associated with age at diagnosis $\geqslant 60$ years (RR 1.40), no family history ( $R R \quad 1.35$ ), white race ( RR 1.25$)$, $\geqslant 13$ years of education (RR 1.49), body mass index $<25.0 \mathrm{~kg} / \mathrm{m}^{2}$ (RR 1.52 ), $\geqslant 13$ years old at menarche (RR 1.42), having had a child (RR $1.34),<25$ years of age at birth of first child (RR 1.41), no use of oral contraceptives in the past 10 years (RR 1.35), ever having smoked (RR 1.56), and natural menopause (RR 1.32). The RR associated with long durations of current or recent use decreased with increasing weight $(\mathrm{p}<0.001$ for trend) and increasing body mass index ( $\mathrm{p}<0.001$ for trend).

\section{Conclusions}

Women who are current or recent users of hormone replacement therapy have an increased risk of breast cancer compared with those who are never users; this risk is greater with increasing duration of use. Women who stop hormone replacement therapy have a reduced risk and, after stopping for $\geqslant 5$ years, have no increase in risk.

Source of funding: Imperial Cancer Research Fund.

For article reprint: Professor V Beral, Secretariat, ICRF Cancer Epidemiology Unit, Gibson Building, Radcliffe Infirmary, Oxford OX2 6HE, UK. Fax +44 (0)1865 310545.

A modified version of this abstract appears in ACP Journal Club 1998 May-Jun.

\section{Commentary}

This timely and long awaited collaborative work will be of interest to many women. Both endogenous and exogenous female hormones have featured in the identification of risk factors for breast cancer. Oral contraceptive use, early menarche, late first pregnancy, late menopause, and nulliparity are known to play some part in altering cell proliferation, differentiation, and atrophy as the concentrations and duration of hormonal excretion vary. ${ }^{1}$

In contrast, HRT has been widely advocated for perimenopausal and postmenopausal women because it has been shown to decrease the incidence of cardiovascular disease, reduce the frequency of osteoporotic fractures, and effectively treat many of the physical and psychological symptoms associated with menopause. In this important overview, the authors dealt with the complexity of the many variant trials through stratification, resulting in a detailed study. Because $80 \%$ of the women mostly used preparations containing oestrogens alone and only 12\% took preparations containing both oestrogen and progestagen, there remains too little information to draw firm conclusions on the impact of current practice in which HRTs are used in combination.

Results of this well done meta-analysis indicate an increased risk of breast cancer among women who take HRT for $\geqslant 5$ years. The risk is related to duration of treatment and seems to diminish within 5 years of stopping HRT. Counselling about these risks must also be placed in the context of what is known about the long term benefits (prevention of coronary events and fractures), and long term risks (such as the possibility of endometrial cancer). ${ }^{2}$ It is noteworthy that these findings are based on observational data. We await the results of the Women's Health Initiative in the US, a trial designed specifically to assess the risks and benefits of long term HRT.

Until results from this trial are available, we need to openly discuss with women the potential benefits and risks of HRT in conjunction with individual concerns and health priorities.

Marilyn Kirshbaum, RN, RGN, MSc Research Sister in Breast Care Greenlea Oncology Unit Huddersfield NHS Trust Huddersfield, West Yorkshire, UK

1 Key TJ, Pike MC. The role of oestrogens and progestagens in the epidemiology and prevention of breast cancer. Eur J Cancer Clin Oncol 1988:24:29-43.

2 LaCroix AW, Burke W. Breast cancer and hormone replacement therapy (commentary). Lancet 1997;350:1042-3. 